\title{
Variations on a theme of Burns and Medvedev
}

\author{
Dan Segal
}

For Avinoam Mann, inspiring colleague and friend

\begin{abstract}
Burns and Medvedev prove in $[\mathrm{BM}]$ that a relatively free pro- $p$ group cannot be $p$-adic analytic unless it is virtually nilpotent. We present a shorter, more conceptual proof, and apply it to deduce analogous results for other categories of groups.
\end{abstract}

Mathematics Subject Classification (2000). Primary 20E10, 20 E18.

Keywords. Relatively free groups, profinite groups.

\section{Introduction}

The following theorem is established in $[\mathrm{BM}]$ :

Theorem 1. A relatively free pro- $p$ group is $p$-adic analytic if and only if it is finitely generated and virtually nilpotent.

The rather technical proof given in $[\mathrm{BM}]$ relies ultimately on a deep theorem of Zelmanov about Engel Lie algebras. The aim of this note is to present a short and relatively easy, or at least more conceptual, proof. An advantage of this approach is that it yields analogous results for other categories of groups.

Before stating these, let us recall that a pro- $p$ group $G$ is $p$-adic analytic if and only if it has finite rank ([DDMS], Chapter 8); the rank of any profinite group $G$ is the least integer $r$ such that every closed subgroup of $G$ can be generated (topologically) by $r$ elements. An abstract group $G$ is said to have rank $r$ if every finitely generated subgroup of $G$ can be generated by $r$ elements, and $r$ is minimal with this property (this is also known as the Mal'cev special rank or Prüfer rank). The upper rank of $G$ is the supremum of the ranks of all finite quotient groups of $G$; it coincides with the rank of the profinite completion $\widehat{G}$ of $G$.

We also need to discuss what is meant by "relatively free". In $[\mathrm{BM}]$, a pro- $p$ group is (in effect) defined to be relatively free if it is of the form $F / R$ where $F$ is 
a free pro- $p$ group and $R$ is a closed fully invariant subgroup; such an $R$ need not, a priori, be a verbal subgroup (it is generated by the values of a family of 'profinite words'). I will call a profinite group $G$ relatively free of finite type in a group variety if $G=F / \overline{\mathcal{V}(F)}$, where $F$ is the free profinite group on some finite set and $\mathcal{V}(F)$ is a verbal subgroup, i.e. the subgroup generated (algebraically) by the values of some family $\mathcal{V}$ of (ordinary) group words (and $\bar{S}$ denotes the closure of a subset $S$ in a profinite group). (In the world of free groups, 'finite type' is traditionally called 'finite rank', a terminology it seems best to avoid in the present context.)

Theorem 2. Let $G$ be a profinite group that is relatively free of finite type in a group variety. Then $G$ has finite rank if and only if $G$ is virtually nilpotent.

For the next variation we recall some material from [RZ], Chapter 2 and Chapter 3. A formation will mean a class $\mathcal{C}$ of finite groups closed w.r.t. forming quotients and finite subdirect products. A pro- $\mathcal{C}$ group is a projective limit of groups in $\mathcal{C}$, or equivalently a profinite group all of whose continuous finite images are in $\mathcal{C}$. For a finite set $X$, the free pro- $\mathcal{C}$ group on $X$ is the pro- $C$ completion of the abstract free group on $X$. We define a relatively free pro- $C$ group of finite type in a group variety as above, replacing 'profinite' by 'pro- $\varrho$ '.

Theorem 3. Let $\mathcal{C}$ be a subgroup-closed formation consisting of soluble groups; assume that, for some prime $p$, $\mathcal{C}$ contains $C_{p} \prec C_{p^{n}}$ for each $n \in \mathbb{N}$. Let $G$ be a relatively free pro-C group of finite type in a group variety. Then $G$ has finite rank if and only if $G$ is virtually nilpotent.

(Here $C_{p} \prec C_{p^{n}}$ denotes the wreath product of a cyclic group of order $p$ by a cyclic group of order $p^{n}$.)

Of course, if $\mathfrak{V}$ is a group variety and $\mathcal{C}$ is a formation then $\mathfrak{V} \cap \mathcal{C}=\mathcal{C}^{*}$ is again a formation, and a pro- $\mathcal{C}$ group relatively free in $\mathfrak{V}$ is the same thing as a free pro- $\ell^{*}$ group; the theorem is stated as it is to show how it generalizes Theorem 1 in the most 'natural' case, that of group varieties (for $\mathcal{C}$ one could take for example all finite soluble groups, or all finite soluble $\pi$-groups where $\pi$ is a non-empty set of primes).

These results about profinite groups will be deduced from an analogous one about abstract groups:

Theorem 4. A finitely generated relatively free group $G$ has finite upper rank if and only if $G / G_{f}$ is virtually nilpotent, where $G_{f}$ denotes the finite residual of $G$. 


\section{The main step}

The 'if' claims in the theorems are well known and elementary. The proofs of the other implications are based on the following observation:

Proposition 1. Let $G$ be (a) a relatively free group, or (b) a relatively free pro- $p$ group. Suppose that $G$ is virtually torsion-free and virtually soluble of finite rank. Then $G$ is virtually nilpotent.

Proof. We may assume that $G$ is relatively free on at least two generators. $G$ has (closed) normal subgroups $N \leq H$ such that $G / H$ is finite, $H / N$ is abelian and $N$ is torsion-free and nilpotent (a well-known consequence of the Lie-Kolchin-Mal'cev theorem, cf. [LR], 5.2.1, 5.2.2). Let $Z_{i}$ denote the $i$ th term of the upper central series of $N$; then each factor $Z_{i} / Z_{i-1}$ is a torsion-free abelian group (resp. pro- $p$ group) of rank at most $r$, the rank of $G$. Writing $Z_{i} / Z_{i-1}$ additively we consider

$$
V_{i}:=\left(Z_{i} / Z_{i-1}\right) \otimes k
$$

as a $k H$-module, where $H$ acts by conjugation and $k=\mathbb{Q}$ in case (a), $k=\mathbb{Q}_{p}$ in case (b). Note that $V_{i}$ is a vector space over $k$ of dimension at most $r$. I claim that for each $i$ and each $h \in H$,

$$
h^{r !} \text { acts unipotently on } V_{i} \text {. }
$$

As $H / N$ is abelian, this will imply that the group $N H^{r !}$ is nilpotent, and the result follows; in case (a) because $\left|G: N H^{r !}\right|$ is finite, in case (b) because $\overline{N H^{r !}}$ is also nilpotent, and has finite index in $G$ (in fact $N H^{r !}$ is already closed, cf. [DDMS], Chapter 1).

To establish the claim, let $a \in Z_{i} / Z_{i-1}$, put $U=a \cdot k\langle h\rangle \leq V_{i}$ and let $\eta \in \operatorname{GL}(U)$ correspond to the action of $h$. As $V_{i}$ is the sum of finitely many modules like $U$, it will suffice to show that $\eta^{r !}$ is unipotent. Now consider the two elements

$$
v=(a, \ldots, a), \quad \gamma=\left(\gamma_{1}, \ldots, \gamma_{n}\right) \in\left(G / Z_{i-1}\right)^{(n)},
$$

the direct product of $n=r+1$ copies of $G / Z_{i-1}$, where $\gamma_{j}=h^{j} Z_{i-1}$ for each $j$. Put $\Delta=\langle v, \gamma\rangle$ in case (a), $\Delta=\overline{\langle v, \gamma\rangle}$ in case (b). Since $G$ is relatively free on at least two generators, there is an epimorphism from $G$ onto $\Delta$, and so $\Delta$ has rank at most $r$. Therefore

$$
W:=\left(\left(Z_{i} / Z_{i-1}\right)^{(n)} \cap \Delta\right) \otimes k
$$

is a $k$-vector space of dimension at most $r$. Evidently

$$
v^{\gamma}=\left(a \eta, a \eta^{2}, \ldots, a \eta^{n}\right) .
$$


Let $f(X)$ denote the characteristic polynomial of the action of $\gamma$ on $W$. Then $f(\gamma)$ annihilates $W$, and as $v \in W$ it follows that for $j=1, \ldots, n$ we have $a \cdot f\left(\eta^{j}\right)=0$, whence $f\left(\eta^{j}\right)=0$. Now let $\lambda$ be an eigenvalue of $\eta$. As $f$ has at most $r<n$ roots, there exist $s$ and $t$ with $1 \leq s<t \leq n$ such that $\lambda^{s}=\lambda^{t}$. Thus $\lambda^{e}=1$ where $e=t-s \leq n-1=r$, and so $\lambda^{r !}=1$.

Thus $\eta^{r !}$ is unipotent as required.

Remark. Essentially the same argument shows that if $G$ is not virtually nilpotent, then the Cartesian power $G^{\mathbb{N}}$ involves as a section the wreath product $C_{\infty}$ ? $C_{\infty}$ of two infinite cyclic groups. Since the group $C_{\infty}<C_{\infty}$ generates the variety of all metabelian groups (see [N], Theorem 22.42), we may infer the following corollary of the proof:

Corollary 1. Let $G$ be a soluble group of finite rank which is virtually torsion-free. If $G$ is not virtually nilpotent then the variety generated by $G$ contains all metabelian groups.

This generalizes an example due to B. H. Neumann, [N], 32.35, which indeed suggested the above proof of Proposition 1.

\section{Pro- $p$ groups}

Let $G=F / R$ be a pro- $p$ group of finite rank, where $F$ is a free pro- $p$ group and $R$ is a fully invariant closed subgroup. Then $G$ is virtually torsion-free and $G$ is a linear group over $\mathbb{Q}_{p}$ ([DDMS], Chapter 4 , Chapter 7). Suppose that $R$ contains a non-trivial verbal subgroup $w(F)$. Then $w(G)=1$, and it follows by a theorem of Platonov (see [W], 10.15) that $G$ is virtually soluble (of course this follows from the well-known 'Tits alternative', but that is a much harder result). Proposition 1 now shows that $G$ is virtually nilpotent (and $G$ is finitely generated because it has finite rank).

Thus Theorem 1 is proved for pro- $p$ groups that are relatively free in a group variety (this case suffices for the interesting application made in [JZ]). The general case will follow from the next lemma:

Lemma 1. Let $F$ be a free pro-p group and let $R$ be a fully invariant closed subgroup of $F$. Suppose that $F / R$ has finite rank. Then $R$ contains $w(F)$ for some non-trivial group word $w$.

Proof. We may assume that $F$ is the free pro- $p$ group on a set $X$ with $|X| \geq 2$ (otherwise we may take $w=[x, y]$ ). Suppose first that $3 \leq|X|<\infty$. Then $F$ is the 
pro- $p$ completion of the abstract free group $\Phi$ on $X$, which we consider as a subgroup of $F$. We have a natural homomorphism

$$
\theta: \operatorname{Aut}(\Phi) \rightarrow \operatorname{Aut}(F) \rightarrow \operatorname{Aut}(F / R)
$$

Now $\operatorname{Aut}(F / R)$ is a linear group over $\mathbb{Q}_{p}$ ([DDMS], Chapter 5 and Chapter 7); but $\operatorname{Aut}(\Phi)$ has no faithful linear representation over any field, by a theorem of Formanek and Procesi [FP]. Thus $\theta$ is not injective. Let $1 \neq \alpha \in \operatorname{ker} \theta$. Then

$$
1 \neq[\Phi, \alpha] \subseteq \Phi \cap[F, \alpha \theta] \subseteq \Phi \cap R
$$

Then for $1 \neq w=w(X) \in[\Phi, \alpha]$ we have $w(F) \subseteq R$ since $R$ is fully invariant in $F$.

Suppose next that $X$ is infinite. In a finitely generated pro- $p$ group, every generating set contains a finite generating set, so $F=R \cdot F(Y)$ where $Y$ is a finite subset of $X$ and $F(Y)$ denotes the subgroup generated (topologically) by $Y$, which is a free pro- $p$ group on $Y$. We may choose $Y$ to have size at least 3. Now $R \cap F(Y)$ is fully invariant in $F(Y)$, hence, by the first case, contains $w(Y)$ for some non-trivial word $w$. As $Y$ is part of a free basis for $F$ it follows that $w(F) \subseteq R$.

Finally we consider the case where $|X|=2$. For any pro- $p$ group $H$, define

$$
R(H)=\overline{\langle R \phi \mid \phi \in \mathscr{H}\rangle}
$$

where $\mathscr{H}$ denotes the set of all morphisms $F \rightarrow H$. Then $R(H)$ is fully invariant in $H$. Now take $H$ to be the free pro- $p$ group on $X \cup Y$ where $Y$ is a non-empty finite set disjoint from $X$. Then $F$ is a subgroup of $H$, and there is a morphism $\pi: H \rightarrow F$ with $Y \pi=\{1\}$ and $\pi_{\mid F}=\operatorname{Id}_{F}$. Thus

$$
\begin{aligned}
F \cap R(H) & =(F \cap R(H)) \pi \\
& \leq R(H) \pi=\overline{\langle R \phi \pi \mid \phi \in \mathscr{H}\rangle}=R
\end{aligned}
$$

since $R \phi \pi \subseteq R$ for each $\phi \in \mathscr{H}$. By the first case, we have $w(H) \subseteq R(H)$ for some non-trivial word $w$; then $w(F) \subseteq F \cap R(H) \subseteq R$.

This completes the proof.

\section{Abstract groups}

Let $G$ be a relatively free group. It is easy to see that $G_{f}$ is a fully invariant subgroup of $G$, so $G / G_{f}$ is again relatively free. Thus to prove Theorem 4 we may replace $G$ by $G / G_{f}$, and so assume that $G$ is residually finite. If also $G$ is finitely generated and has finite upper rank, then $G$ is virtually soluble of finite rank, by Theorem A of [MS] (cf. [LS], Theorem 5.2). Such a group which is finitely generated and residually finite is also virtually torsion-free (see [LR], 5.2.1, 10.5.3). Now Proposition 1 shows that $G$ is virtually nilpotent, and Theorem 4 follows. 


\section{Profinite groups}

Let $\mathcal{C}$ be a formation, let $F$ be the free pro- $\mathcal{C}$ group on a finite set $X$, and put $G=F / \overline{\mathcal{V}(F)}$ where $\mathcal{V}$ is a set of group words. Let $\Phi$ be the abstract free group on $X$, and put $\Gamma=\Phi / \mathcal{V}(\Phi)$.

We consider $\Phi$ as a dense subgroup of $F=\widehat{\Phi}_{\ell}$, the pro- $\mathcal{C}$ completion of $\Phi$. Suppose that $N$ is an open normal subgroup of $F$. Then $F=N \Phi$, so $\mathcal{V}(F) \subseteq N \mathcal{V}(\Phi)$. As the intersection of all such sets $N \mathcal{V}(\Phi)$ is equal to the closure of $\mathcal{V}(\Phi)$ in $F$, it follows that $\mathcal{V}(F) \subseteq \overline{\mathcal{V}(\Phi)}$ and hence that $\overline{\mathcal{V}(F)}=\overline{\mathcal{V}(\Phi)}$.

Now let $\Gamma_{\ell}$ denote the $\mathcal{C}$-residual of $\Gamma$ and put $\Gamma_{0}=\Gamma / \Gamma_{\ell}$; thus $\Gamma_{\ell}$ is the kernel of the natural map $\Gamma \rightarrow \hat{\Gamma}_{e}$, and we have

$$
\widehat{\left(\Gamma_{0}\right)_{e}}=\hat{\Gamma}_{\mathcal{C}} \cong \widehat{\Phi}_{\mathcal{C}} / \overline{\mathcal{V}(\Phi)}=F / \overline{\mathcal{V}(F)}=G .
$$

Assuming that $G$ has finite rank $r$ as a profinite group, it follows that every $\mathcal{C}$ quotient of $\Gamma$ has rank at most $r$.

Case 1. Suppose that $\mathcal{C}$ consists of all finite groups. Then $\Gamma$ has upper rank $r$, and Theorem 4 shows that $\Gamma / \Gamma_{f}=\Gamma_{0}$ is virtually nilpotent. Therefore so is $\hat{\Gamma}_{0}=$ $\widehat{\left(\Gamma_{0}\right)} \mathcal{C} \cong G$, and Theorem 2 follows.

Case 2. Suppose now that $\mathcal{C}$ satisfies the hypotheses of Theorem 3. If $|X| \leq 1$ then $G$ is abelian and there is nothing to prove, so we assume that $|X| \geq 2$. Then $C_{p} \prec C_{p^{n}}$ is a $\mathcal{C}$-quotient of $\Phi$ for each $n$; but it cannot be a quotient of $\Gamma$ if $p^{n}>r$, consequently $\mathcal{V}\left(C_{p} \prec C_{p^{n}}\right) \neq 1$ for large $n$. In particular, $\mathcal{V}$ is not trivial.

Now $\Gamma_{0}$ is residually (finite soluble of rank at most $r$ ). It follows by the main theorem of [S] that $\Gamma_{0}$ is an extension of a nilpotent group by a subdirect product of finitely many linear groups. As $\mathcal{V}\left(\Gamma_{0}\right)=1$, Platonov's theorem [W], 10.15 (cited above) implies that each of these linear groups is virtually soluble, and hence that $\Gamma_{0}$ has a soluble normal subgroup $\Gamma_{1}$ of finite index. The second part of the theorem of $[\mathrm{S}]$ now shows that $\Gamma_{1}$ is virtually nilpotent-by-abelian, and we conclude that $\Gamma_{0}$ is virtually nilpotent-by-abelian. Below I will prove the following:

Lemma 2. Let $H$ be a finitely generated group and $N$ a torsion-free nilpotent normal subgroup such that $H / N$ is abelian. Then either $H$ has finite rank or $H$ has a section isomorphic to $C_{\infty}$ 々 $C_{\infty}$.

Lemma 3. Let $T$ be a periodic nilpotent group and $\mathcal{X}$ a filter base of normal subgroups. If the members of $\mathcal{X}$ intersect in 1 and the quotients $T / K$ with $K \in \mathcal{X}$ have bounded finite ranks then $T$ has finite rank.

(To say that $\mathcal{X}$ is a filter base means that the intersection of any two members of $\mathcal{X}$ contains a member of $\mathcal{X}$.) 
To continue with the proof of Theorem 3 , let $\Gamma_{2} \geq \Gamma_{3}$ be normal subgroups of $\Gamma_{0}$ such that $\Gamma_{0} / \Gamma_{2}$ is finite, $\Gamma_{2} / \Gamma_{3}$ is abelian and $\Gamma_{3}$ is nilpotent. Let $T$ be the torsion subgroup of $\Gamma_{3}$ and put $H=\Gamma_{2} / T, N=\Gamma_{3} / T$. Since $\mathcal{V}\left(\Gamma_{0}\right)=1$ while $\left.\mathcal{V}\left(C_{p}\right\rangle C_{p^{n}}\right) \neq 1$ for large $n$, the group $C_{\infty} \prec C_{\infty}$ cannot be a section of $H$, so $H$ has finite rank by Lemma 2. Now let

$$
\mathcal{X}=\left\{M \cap T \mid M \triangleleft \Gamma_{0}, \quad \Gamma_{0} / M \in \ell\right\} .
$$

Then $\mathcal{X}$ satisfies the hypotheses of Lemma 3, and we may infer that $T$ has finite rank.

It follows that $\Gamma_{0}$ has finite rank. As remarked in Section 4, residual finiteness implies that $\Gamma_{0}$ is virtually torsion-free. Noting that $\zeta$ is a subgroup-closed formation, it is easy to see that $\Gamma_{e}$ is fully invariant in $\Gamma$, and hence that $\Gamma_{0}=\Gamma / \Gamma_{e}$ is a relatively free group. Applying Proposition 1 (a) we deduce that $\Gamma_{0}$ is virtually nilpotent. Therefore so is $\widehat{\left(\Gamma_{0}\right)} \mathcal{e} \cong G$ and Theorem 3 is proved.

Proof of Lemma 2. Assuming that $C_{\infty} \prec C_{\infty}$ is not a section of $H$, we need to show that $N$ has finite rank.

Suppose to begin with that $N$ is abelian. The result then follows from the main theorem of $[\mathrm{K}]$; but let me sketch the proof of this easy special case. We consider $N$ as an additively-written module for $H / N=\left\langle x_{1}, \ldots, x_{n}\right\rangle$. Since $H$ is finitely generated and $H / N$ is finitely presented, $N$ is finitely generated as a module, say $N=a_{1} \mathbb{Z}(H / N)+\cdots+a_{r} \mathbb{Z}(H / N)$. If $v \in N$ then each $v \cdot \mathbb{Z}\left\langle x_{i}\right\rangle$ has finite rank (otherwise $\left\langle v, \widetilde{x_{i}}\right\rangle \cong C_{\infty}<C_{\infty}$ where $\widetilde{x_{i}}$ is a pre-image of $x_{i}$ in $H$ ). Now fix $k \leq r$ and suppose that $a_{k} \cdot \mathbb{Z}\left\langle x_{1}, \ldots, x_{m}\right\rangle$ has finite rank for some $m<n$; let $\left\{v_{1}, \ldots, v_{d}\right\}$ be a maximal linearly independent subset. Then $\sum_{j=1}^{d} v_{j} \cdot \mathbb{Z}\left\langle x_{m+1}\right\rangle$ has finite rank and contains a maximal linearly independent subset of $a_{k} \cdot \mathbb{Z}\left\langle x_{1}, \ldots, x_{m}, x_{m+1}\right\rangle$, which therefore has finite rank. It follows by induction that $a_{k} \mathbb{Z}(H / N)$ has finite rank. As this holds for each $k$ we see that $N$ has finite rank.

In the general case, we may infer that $N / T$ has finite rank where $T / \gamma_{2}(N)$ is the torsion subgroup of $N^{a b}=N / \gamma_{2}(N)$. Each lower central factor $\gamma_{i}(N) / \gamma_{i+1}(N)$ is an epimorphic image of a tensor power of $N^{a b}$ ([LR], 1.2.12), so the lower central series of $N$ is a finite filtration with each factor abelian of finite torsion-free rank. Each upper central factor $Z_{j}(N) / Z_{j-1}(N)$ of $N$ then inherits such a filtration. But $Z_{j}(N) / Z_{j-1}(N)$ is torsion-free ([LR], 2.3.8), and therefore has finite rank. It follows that $N$ has finite rank.

Proof of Lemma 3. Let $Z$ be the centre of $T$. Elementary arguments show that $T / Z$ and its family of normal subgroups $\{K Z / Z \mid K \in \mathcal{X}\}$ satisfy the hypotheses of the lemma; arguing by induction on the nilpotency class of $T$, we may suppose that $T / Z$ has finite rank $s$, say. Now let $r$ be an upper bound for the ranks of the quotients $T / K$ with $K \in \mathcal{X}$, and let $E$ be an elementary abelian $p$-subgroup of $Z$. Then $|E: E \cap K| \leq p^{r}$ for each $K \in \mathcal{X}$, and the hypotheses on $\mathcal{X}$ then imply that 
$|E| \leq p^{r}$. As $Z$ is a periodic abelian group it follows that $Z$ rank at most $r$. We conclude that $T$ has rank at most $r+s$.

\section{References}

[BM] R. G. Burns and Y.Medvedev, Analytic relatively free pro- $p$ groups. J. Group Theory 7 (2004), 533-541. Zbl 1073.20021 MR 2080450

[DDMS] J. D. Dixon, M. P. F. du Sautoy, A. Mann, and D. Segal, Analytic pro- $p$ groups. 2nd ed., Cambridge Stud. Adv. Math. 61, Cambridge University Press, Cambridge 1999. Zbl 0934.20001 MR 1720368

[FP] E. Formanek and C. Procesi, The automorphism group of a free group is not linear. J. Algebra 149 (1992), 494-499. Zbl 0780.20023 MR 1172442

[JZ] A. Jaikin-Zapirain, On the verbal width of finitely generated pro- $p$ groups. Rev. Mat. Iberoamericana, to appear. http://www.uam.es/personal_pdi/ciencias/ajaikin/preprints/verbal.pdf

[K] P. H. Kropholler, On finitely generated soluble groups with no large wreath product sections. Proc. London Math. Soc. (3) 49 (1984), 155-169. Zbl 0537.20013 MR 743376

[LR] J. C. Lennox and D. J. S. Robinson, The theory of infinite soluble groups. Oxford Math. Monographs, The Clarendon Press, Oxford 2004. Zbl 1059.20001 MR 2093872

[LS] A. Lubotzky and D. Segal, Subgroup growth. Progr. Math. 212, Birkhäuser, Basel 2003. Zbl 1071.20033 MR 1978431

[MS] A. Mann and D. Segal, Uniform finiteness conditions in residually finite groups. Proc. London Math. Soc. (3) 61 (1990), 529-545. Zbl 0722.20021 MR 1069514

[N] H. Neumann, Varieties of groups. Ergeb. Math. Grenzgeb. 37, Springer-Verlag, Berlin 1967. Zbl 0251.20001 MR 0215899

[RZ] L. Ribes and P. Zalesskii, Profinite groups. Ergeb. Math. Grenzgeb. (3) 40, SpringerVerlag, Berlin 2000. Zbl 0949.20017 MR 1775104

[S] D. Segal, A footnote on residually finite groups. Israel J. Math. 94 (1996), 1-5. Zbl 0849.20017 MR 1394563

[W] B. A. F. Wehrfritz, Infinite linear groups. Ergeb. Math. Grenzgeb. 76, Springer-Verlag, New York 1973. Zbl 0261.20038 MR 0335656

Received November 30, 2006

D. Segal, All Souls College, Oxford OX1 4AL, UK

E-mail: dan.segal@all-souls.ox.ac.uk 